\title{
Nucleon form factors near the physical point in 2+1 flavor QCD
}

\author{
K.-I. Ishikawa ${ }^{a, b}$, Y. Kuramashi ${ }^{* c, b}$, N. Tsukamoto ${ }^{d}$, S. Sasaki ${ }^{d, e}$, T. Yamazaki ${ }^{f, b, c}$, and \\ A. Ukawa ${ }^{b}$ for PACS Collaboration \\ ${ }^{a}$ Graduate School of Science, Hiroshima University, Higashi-Hiroshima, Hiroshima 739-8526, \\ Japan \\ ${ }^{b}$ RIKEN Advanced Institute for Computational Science, Kobe, Hyogo 650-0047, Japan \\ ${ }^{c}$ Center for Computational Sciences, University of Tsukuba, Tsukuba, Ibaraki 305-8577, Japan \\ ${ }^{d}$ Department of Physics, Tohoku University, Sendai 980-8578, Japan \\ ${ }^{e}$ Theoretical Research Division, Nishina Center, RIKEN, Wako 351-0198, Japan \\ ${ }^{f}$ Graduate School of Pure and Applied Sciences, University of Tsukuba, Tsukuba, Ibaraki, \\ 305-8571, Japan \\ E-mail: kuramasi@het.ph.tsukuba.ac.jp
}

\begin{abstract}
We present preliminary results for nucleon form factors including the axial charge and the isovector electric radius in $2+1$ flavor QCD at almost the physical pion mass on a $96^{4}$ lattice with the lattice spacing of $0.085 \mathrm{fm}$. The configurations are generated with the stout-smeared $O(a)$ improved Wilson quark action and the Iwasaki gauge action at $\beta=1.82$. A large spatial volume of $(8.1 \mathrm{fm})^{3}$ allows us to investigate the form factors at small momentum transfer region. We discuss analyses of the momentum dependence of the form factors with the z-expansion.
\end{abstract}

34th annual International Symposium on Lattice Field Theory

24-30 July 2016

University of Southampton, UK

\footnotetext{
* Speaker.
} 


\section{Introduction}

Nucleon form factors are good probes to investigate the internal structure of the nucleon. Much effort has been devoted to calculate them with lattice QCD since 1980's. Unfortunately, the current situation is that we are still struggling for reproducing the well-established experimental results, e.g., the axial vector coupling and the electric charge radius. This means that we have not yet achieved proper treatment of a single hadron in lattice QCD calculation. In this report we present the results of the nucleon form factors measured on an $(8.1 \mathrm{fm})^{4}$ lattice at almost the physical point in $2+1$ flavor QCD. Thanks to the large spatial volume we can access to the small momentum transfer region up to $152 \mathrm{MeV}$. The momentum dependence of the nucleon form factors is examined based on the dipole form, the simple Taylor expansion and the $z$-expansion. We perform a detailed comparison between three analyses.

\section{Simulation details}

We employ the $2+1$ flavor QCD gauge configurations generated with the stout-smeared $O(a)$ improved Wilson-clover quark action and the Iwasaki gauge action[1] on a $96^{4}$ lattice at $\beta=$ $1.82[2]$. The value of the improvement coefficient is nonperturbatively determined as $c_{\mathrm{SW}}=1.11$ with the Schrödinger functional scheme[3]. The lattice spacing is about $0.085 \mathrm{fm}$ so that the physical lattice size amounts to $(8.1 \mathrm{fm})^{4}$. The pion mass at the simulation point is about $145 \mathrm{MeV}$, which is fairly close to the physical point. The results for the hadron spectrum and other physical quantities are already presented in Ref. [2].

The nucleon form factors are extracted from the three-point functions consisting of the nucleon source and sink operators and the local vector or axial vector currents:

$$
\begin{aligned}
& C_{V_{\alpha}}^{\mathscr{P}}\left(t, \vec{p}^{\prime}, \vec{p}\right)=\frac{1}{4} \operatorname{Tr}\left\{\mathscr{P}\left\langle N\left(t_{\mathrm{sink}}, \vec{p}^{\prime}\right) V_{\alpha}(t, \vec{q}) \bar{N}\left(t_{\mathrm{src}},-\vec{p}\right)\right\rangle\right\} \\
& C_{A_{\alpha}}^{\mathscr{P}}\left(t, \vec{p}^{\prime}, \vec{p}\right)=\frac{1}{4} \operatorname{Tr}\left\{\mathscr{P}\left\langle N\left(t_{\mathrm{sink}}, \vec{p}^{\prime}\right) A_{\alpha}(t, \vec{q}) \bar{N}\left(t_{\mathrm{src}},-\vec{p}\right)\right\rangle\right\}
\end{aligned}
$$

with $\mathscr{P}$ appropriate projection operators to extract the form factors and $\vec{q}=\vec{p}-\vec{p}^{\prime}$ the threedimensional momentum transfer. We consider only the rest frame of the final state with $\vec{p}^{\prime}=\overrightarrow{0}$, which leads to $Q^{2}=2 M_{N}\left(E_{N}(\vec{q})-M_{N}\right)$ for the squared four-momentum transfer. We calculate only the connected diagrams to concentrate on the isovector part of the nucleon form factors. The three point functions are constructed with the sequential source method. The currents are moved between the nucleon source and sink operators, both of which are exponentially smeared in the Coulomb gauge, separated by 15 time slices. We employ 9 cases of momentum transfer $\vec{q}=\pi / 48 \times \vec{n}$ with $|\vec{n}|^{2} \leq 9$ in Table1. The minimum momentum transfer is about $152 \mathrm{MeV}$, which is so small as the pion mass. A difference between the results for Q8 and Q9 cases could probe the possible lattice discretization error. All the results presented in this report are preliminarily obtained with 146 configurations performing 64 measurements of the three-point functions on each configuration. We are now increasing the statistics. 


\begin{tabular}{c|ccccccccc}
\hline label & Q1 & Q2 & Q3 & Q4 & Q5 & Q6 & Q7 & Q8 & Q9 \\
\hline$\vec{n}$ & $(1,0,0)$ & $(1,1,0)$ & $(1,1,1)$ & $(2,0,0)$ & $(2,1,0)$ & $(2,1,1)$ & $(2,2,0)$ & $(3,0,0)$ & $(2,2,1)$ \\
$|\vec{n}|^{2}$ & 1 & 2 & 3 & 4 & 5 & 6 & 8 & 9 & 9 \\
degeneracy & 6 & 12 & 8 & 6 & 24 & 24 & 12 & 6 & 24 \\
\hline
\end{tabular}

Table 1: Choices for momentum transfer $\vec{q}=\pi / 48 \times \vec{n}$. The bottom raw denote the degeneracy of $\vec{n}$ due to the permutation symmetry between $\pm x, \pm y, \pm z$ directions.

\section{Nucleon two-point functions}

Let us first investigate the nucleon rest mass and the dispersion relation, which are obtained from the nucleon two-point functions. In Fig. 1 1 we plot the nucleon effective mass for two cases: Smear-local denotes the nucleon two-point function with the smeared source and the local sink operators and smear-smear for the smeared source and the smeared sink ones. We observe that the smear-local case shows good plateau for $t \geq 6$. On the other hand, the signal becomes noisier for the smear-smear case. Figure 2 shows a check of the dispersion relation for the nucleon with the choice of 9 cases of spatial momenta in Table 1. We find that the relativistic continuum dispersion relation is well satisfied up to $|\vec{p}|^{2}=(\pi / 48)^{2} \times 9$.

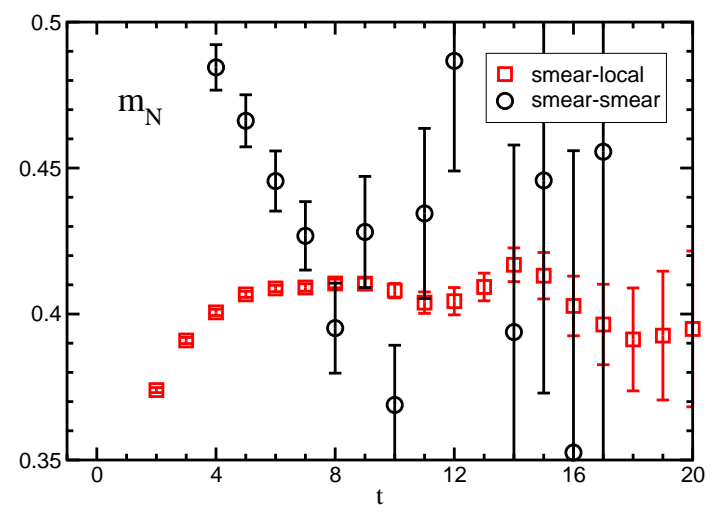

Figure 1: Effective mass for the nucleon.

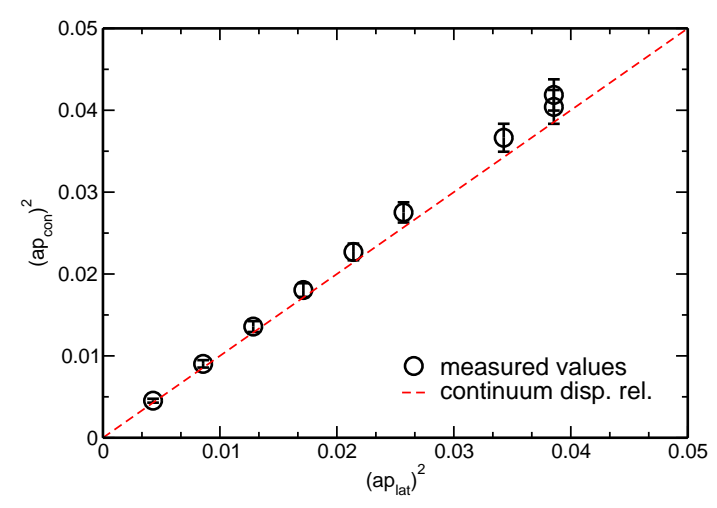

Figure 2: Dispersion relation for the nucleon. $p_{\text {con }}^{2}=\left\{E_{N}\left(\vec{n}^{2}\right)\right\}^{2}-\left\{E_{N}\left(\overrightarrow{0}^{2}\right)\right\}^{2}$ vs. $p_{\text {lat }}^{2}=$ $(\pi / 48)^{2} \times \vec{n}^{2}$.

\section{Nucleon three-point function with zero momentum transfer}

We can evaluate the renormalization factor of the local vector current $Z_{V}$ and the axial charge $g_{A}$ using the three-point functions with zero momentum transfer $\vec{q}=\overrightarrow{0}$. The choice of $\mathscr{P}=(1+$ $\left.\gamma_{4}\right) / 2$ in Eq. 2.1) provides us the Dirac form factor $F_{1}\left(Q^{2}=0\right)=1 / Z_{V}$. Figure 3 plots the result of $Z_{V}$, which shows a good plateau in the time region of $2 \leq t \leq 14$. The fit result with one-sigma error band denoted by three solid lines shows a good consistency with the value of $Z_{V}$ (red line) obtained by the Schrödinger functional (SF) scheme at the vanishing PCAC quark mass [4]. We also draw the value of $Z_{A}$ with the SF scheme for comparison in the same figure. The difference between $Z_{V}$ and $Z_{A}$ is $1.5 \%$, which indicates a fairly small chiral symmetry breaking effects. 
The axial charge $g_{A}$ plotted in Fig. (4) is extracted by choosing $\mathscr{P}=\left(1+\gamma_{4}\right) \gamma_{5} \gamma_{3} / 2$ in Eq. (2.2). The local axial vector current is renormalized with the value of $Z_{A}$ obtained with the SF scheme shown in Fig. 3 The value of $g_{A}$ is obtained from the ratio of the nucleon three-point function divided by the nucleon two-point function. We may have two choices for the denominator: One is the measured value of $\langle N(t) \bar{N}(0)\rangle$ and the other is the single exponential form $A \exp \left(-m_{N} t\right)$ with $A$ and $m_{N}$ the fit results of $\langle N(t) \bar{N}(0)\rangle$. We find that the latter case shows a better agreement with the experimental value. The difference between two cases, which should be a systematic error in this calculation, is expected to diminish as the statistics is increased.

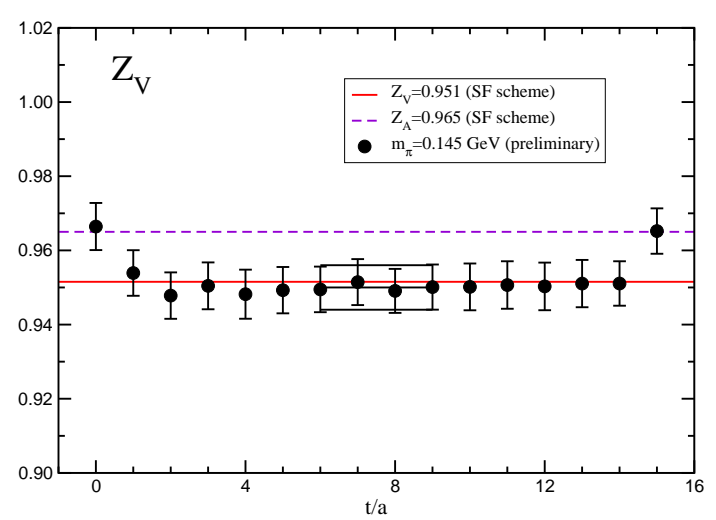

Figure 3: Renormalization factor of the local vector current determined by $Z_{V}=1 / F_{1}(0)$.

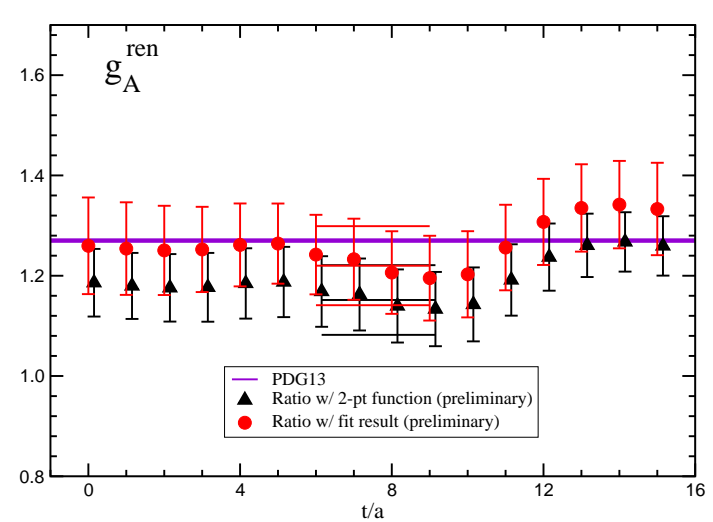

Figure 4: Renormalized axial charge with $Z_{A}=$ $0.9650(68)(95)$ in SF scheme.

\section{Nucleon form factors}

\section{1 $Q^{2}$ dependence of vector form factors}

The Dirac and Pauli form factors $F_{1}\left(Q^{2}\right)$ and $F_{2}\left(Q^{2}\right)$ are related to the electric and magnetic Sachs form factors as

$$
\begin{aligned}
& G_{E}\left(Q^{2}\right)=F_{1}\left(Q^{2}\right)-\frac{Q^{2}}{4 M_{N}^{2}} F_{2}\left(Q^{2}\right), \\
& G_{M}\left(Q^{2}\right)=F_{1}\left(Q^{2}\right)+F_{2}\left(Q^{2}\right) .
\end{aligned}
$$

The isovector form factor is given by a difference between the proton and neutron form factors as $G_{E / M}^{\mathrm{v}}=G_{E / M}^{p}\left(Q^{2}\right)-G_{E / M}^{n}\left(Q^{2}\right) \llbracket 5$. The $Q^{2}$ dependence of $G_{E}$ and $G_{M}$ are plotted in Figs. [5 and 6 respectively, together with the experimental curves. We observe that $G_{E}$ shows good agreement with experimental curve, especially, for low $Q^{2}$. This feature suggests that our results successfully reproduce experimental value for the electric mean squared radius $\left\langle r_{E}^{2}\right\rangle$ which is defined by

$$
\left\langle r_{E}^{2}\right\rangle=-\left.6 \frac{d G_{E}}{d Q^{2}}\right|_{Q^{2}=0}
$$

On the other hand, noisier signals for $G_{M}$ would hinder us from the precise determination of the magnetic mean squared radius $\left\langle r_{M}^{2}\right\rangle$. 


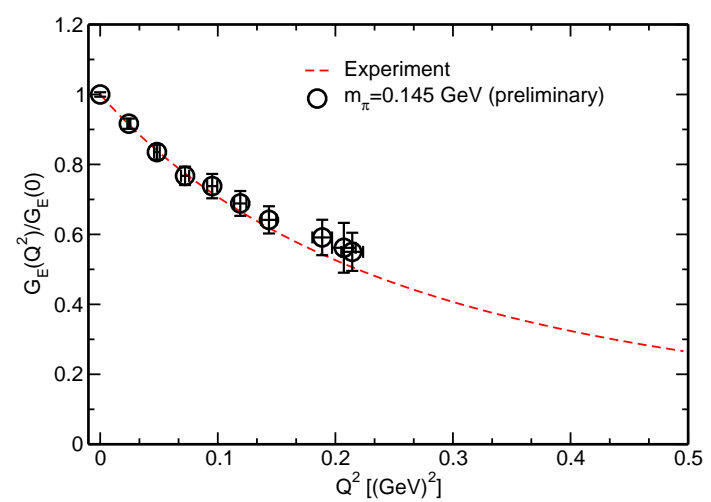

Figure 5: $Q^{2}$ dependence of the isovector electric form factor $G_{E}$. The experimental curve is given by a dipole form with the root mean squared radius of $0.939(5) \mathrm{fm}$.

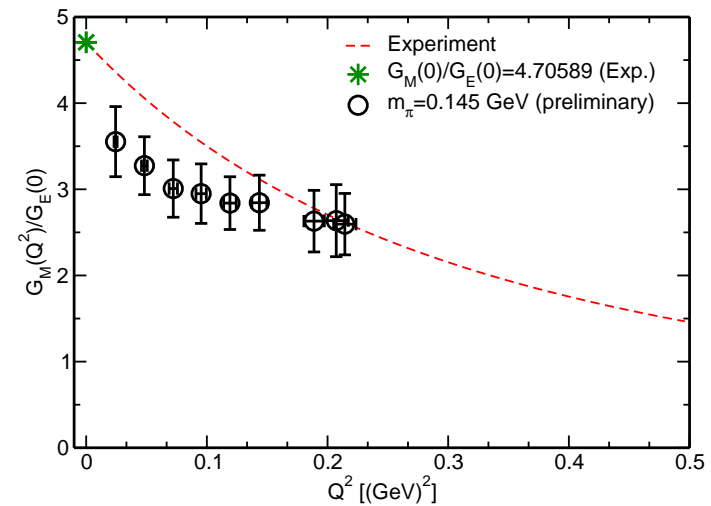

Figure 6: $Q^{2}$ dependence of the isovector magnetic form factor $G_{M}$. The experimental curve is given by a dipole form with the root mean squared radius of $0.862(14) \mathrm{fm}$.

\subsection{Analysis with $z$-expansion}

We first make conventional analyses of the $Q^{2}$ dependence of the electric form factors based on the dipole and quadratic forms. Figure 7 presents the fit results for $G_{E}\left(Q^{2}\right)$ employing the following fit functions:

$$
\begin{aligned}
G_{E}^{d}\left(Q^{2}\right) & =\frac{a_{0}}{\left(1+a_{1} Q^{2}\right)^{2}}, \\
G_{E}^{q}\left(Q^{2}\right) & =d_{0}+d_{2} Q^{2}+d_{4} Q^{4},
\end{aligned}
$$

both of which describe all the data reasonably. The electric root mean squared radius is determined to be $\sqrt{\left\langle r_{E}^{2}\right\rangle}=\sqrt{-12 a_{1}}=0.906(71)$ fm from the dipole fit and $\sqrt{\left\langle r_{E}^{2}\right\rangle}=\sqrt{-6 d_{2} / d_{0}}=0.917(68)$ $\mathrm{fm}$ from the quadratic fit. These values are consistent with the experimental results: 0.9391(58) fm from $e p$ scattering and $0.9073(13) \mathrm{fm}$ from $\mu-\mathrm{H}$ atom spectroscopy 6 .

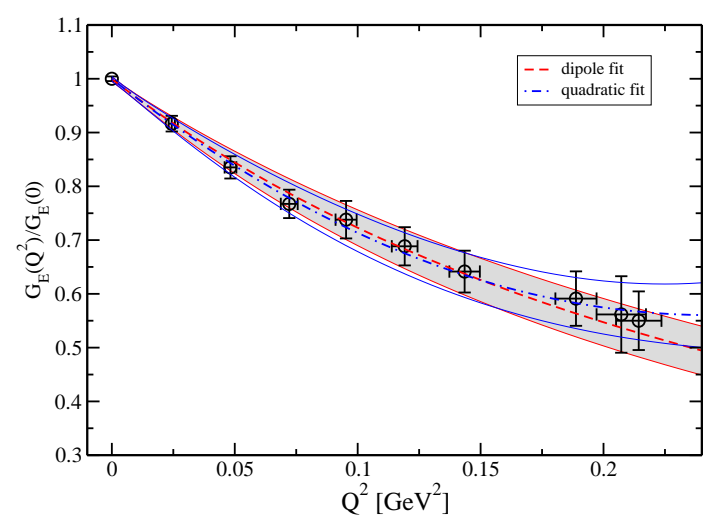

Figure 7: Results for dipole and quadratic fits of $G_{E}$ using all 10 data points.

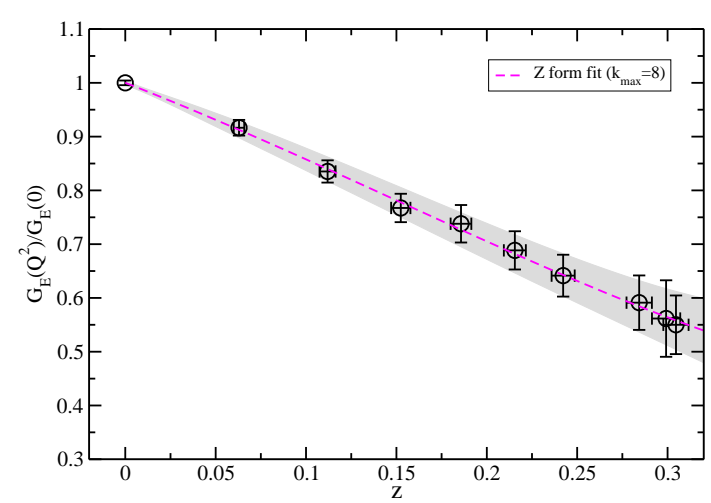

Figure 8: Results for z-expansion fit of $G_{E}$ with $t_{0}=0$ and $k_{\max }=8$ using all 10 data points.

The dipole and quadratic fits give reasonable values for the electric root mean squared radius. However, it is well known that they have clear defects: The dipole fit is model-dependent and 
the Taylor expansion in terms of $Q^{2}$ may be valid only for $\left|Q^{2}\right|<t_{\text {cut }}=4 m_{\pi}^{2}$. A possible way to overcome these defects is the use of the $z$-expansion: $G_{E}(z)=\sum_{k=0}^{k_{\max }} c_{k} z\left(Q^{2}\right)^{k}$, where $z\left(Q^{2}\right)$ is a conformal mapping of the cut plane $Q^{2}<-4 m_{\pi}^{2}$ to the unit circle $|z|=1$ with

$$
z\left(Q^{2}\right)=\frac{\sqrt{t_{\mathrm{cut}}+Q^{2}}-\sqrt{t_{\mathrm{cut}}-t_{0}}}{\sqrt{t_{\mathrm{cut}}+Q^{2}}+\sqrt{t_{\mathrm{cut}}-t_{0}}}
$$

with $t_{\text {cut }}=4 m_{\pi}^{2}[7$. There are three major advantages in this method. Firstly, we can perform a model independent analysis, which is in contrast to the dipole fit. Secondly, analyticity is assured within the unit circle. Thirdly, the constraint $\sum_{k}|| c_{k} \|<\infty$ allows us to expect good convergence for the coefficient $c_{k}$. Figure 8 shows the fit results for $G_{E}\left(Q^{2}\right)$ with $k_{\max }=8$ and $t_{0}=0$ in the z-expansion. It is clear that the curvature becomes smaller in $z$ variable than $Q^{2}$ variable. We investigate the convergence behavior of the coefficients both in the Taylor expansion and the $z$-expansion. Figure 9 plots the magnitude of the coefficient $\left|c_{k}\right|$ for the Taylor expansion: $Q\left(k_{\max }\right) \equiv \sum_{k=0}^{k_{\max }} c_{k}\left(Q^{2} / t_{\text {cut }}\right)^{k}$ with the choices of $k_{\max }=2, \cdots, 8$. Although $\left|c_{0}\right|$ and $\left|c_{1}\right|$ give dominant contributions, $\left|c_{n+1} / c_{n}\right|<1$ is not necessarily satisfied for $n \geq 2$. In Fig. 10 we find different convergence behavior for the $z$-expansion: $z\left(k_{\max }\right) \equiv \sum_{k=0}^{k_{\max }} c_{k} z^{k}$ with $k_{\max }=2, \cdots, 8$. Coefficients are fairly stable for $k_{\max } \leq 4$ and $\left|c_{n+1} / c_{n}\right|<1$ is always satisfied for $n \geq 3$. The electric root mean squared radius is determined to be $\sqrt{\left\langle r_{E}^{2}\right\rangle}=\sqrt{-6\left(c_{1} / c_{0}\right) /\left(4 t_{\text {cut }}\right)}=0.950(123) \mathrm{fm}$ in the $z$-expansion with $t_{0}=0$ and $k_{\max }=8$. This value is consistent with the experimental values from $e p$ scattering and $\mu-\mathrm{H}$ atom spectroscopy, though further statistics is required to resolve the experimental puzzle on the proton size.

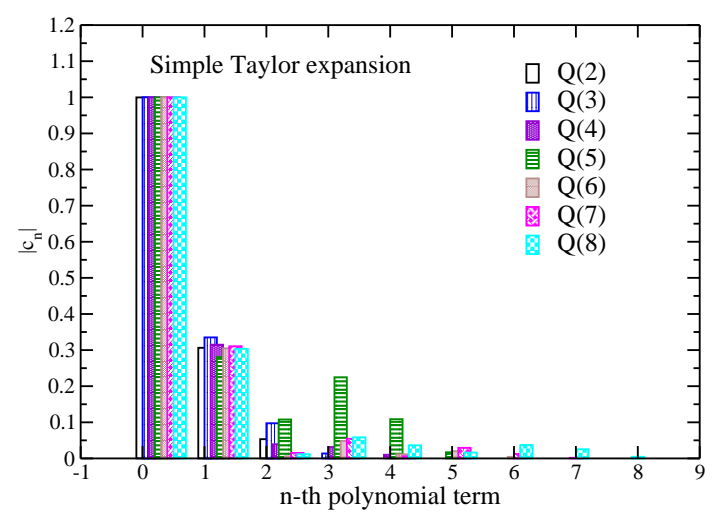

Figure 9: Coefficients of simple Taylor expansion for the electric form factor $G_{E}$.

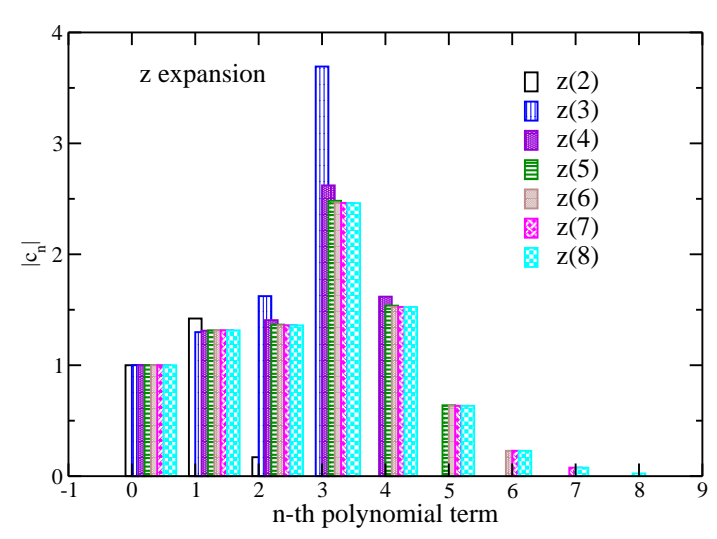

Figure 10: Coefficients of z-expansion for the electric form factor $G_{E}$.

Finally we briefly discuss the analyses of the magnetic form factor $G_{M}\left(Q^{2}\right)$. We show the fit results of $G_{M}\left(Q^{2}\right)$ with the dipole and quadratic functions in Fig. 111 and those with the $z$-expansion $\left(t_{0}=0\right)$ in Fig. 12. All the fits reasonably describe the 9 data points with $\chi^{2} /$ dof $<1$. We have checked that the convergence properties are quite similar to the case of the electric form factor both for the Taylor expansion and the $z$-expansion. The values of the magnetic moment are 3.52(42) for the dipole fit, 3.71(46) for the quadratic fit, and 4.24(70) for the $z$-expansion with $t_{0}=0$ and $k_{\max }=7$. We find that the $z$-expansion gives a consistent result with the experimental value of 4.70589 . 


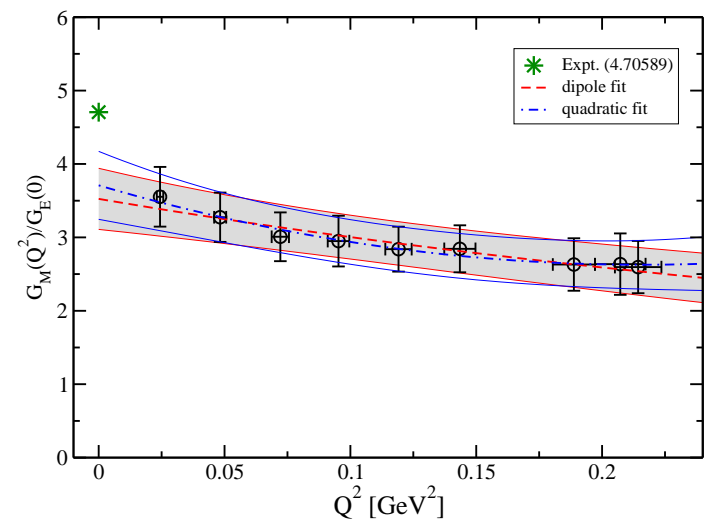

Figure 11: Results for dipole and quadratic fits of $G_{M}$ using all 9 data points.

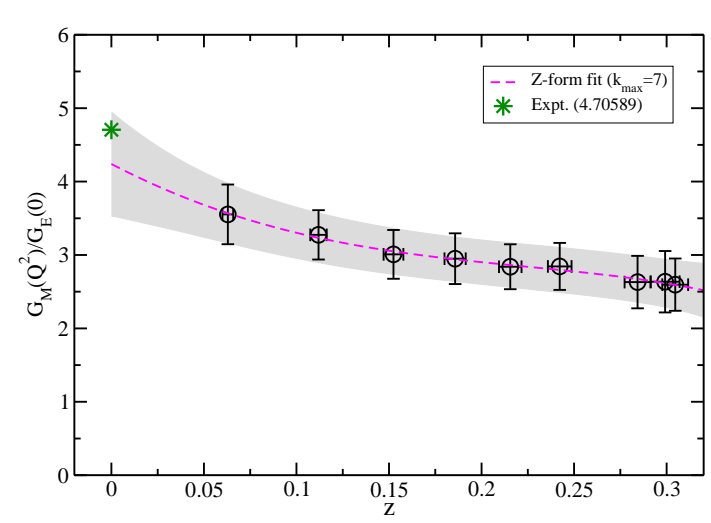

Figure 12: Results for $z$-expansion fit of $G_{M}$ with $t_{0}=0$ and $k_{\max }=7$ using all 9 data points.

\section{Acknowledgments}

Numerical calculations for the present work have been carried out on the FX10 supercomputer system at Information Technology Center of the University of Tokyo, on the HA-PACS and COMA cluster systems under the "Interdisciplinary Computational Science Program" of Center for Computational Science at University of Tsukuba, on HOKUSAI GreatWave at Advanced Center for Computing and Communication of RIKEN, and on the computer facilities of the Research Institute for Information Technology of Kyushu University. This research used computational resources of the HPCI system provided by Information Technology Center of the University of Tokyo, Institute for Information Management and Communication of Kyoto University, the Information Technology Center of Nagoya University, and RIKEN Advanced Institute for Computational Science through the HPCI System Research Project (Project ID: hp120281, hp130023, hp140209, hp140155, hp150135, hp160125). We thank the colleagues in the PACS Collaboration for helpful discussions and providing us the code used in this work. This work is supported in part by MEXT SPIRE Field 5, and also by Grants-in-Aid for Scientific Research from the Ministry of Education, Culture, Sports, Science and Technology (Nos. 22244018, 25800138, 16H06002).

\section{References}

[1] Y. Iwasaki, preprint, UTHEP-118 (Dec. 1983), unpublished.

[2] PACS Collaboration: K.-I. Ishikawa et al., PoS LATTICE 2015, 075 (2015).

[3] Y. Taniguchi, PoS LATTICE 2012, 236 (2012).

[4] PACS Collaboration: K.-I. Ishikawa et al., PoS LATTICE 2015, 271 (2015).

[5] S. Sasaki and T. Yamazaki, Phys. Rev. D78, 014510 (2008).

[6] C. Patrignani et al. (Particle Data Group), Chinese Physics C, 40, 100001 (2016).

[7] C. G. Boyd, B. Grinstein, and R. F. Lebed, Phys. Lett. B353, 306 (1995); R. J. Hill and G. Paz, Phys. Rev. D82, 113005 (2010). 